\title{
Multimodality Imaging of Alzheimer Disease and Other Neurodegenerative Dementias
}

\author{
Ilya M. Nasrallah and David A. Wolk \\ Hospital of the University of Pennsylvania, University of Pennsylvania, Philadelphia, Pennsylvania
}

\begin{abstract}
Learning Objectives: On successful completion of this activity, participants should be able to (1) describe typical MR imaging, ${ }^{18} \mathrm{~F}-\mathrm{FDG}$ PET, and amyloid PET findings in neurodegenerative dementia, including Alzheimer disease, FTLD, and DLB; (2) discuss the roles of MR imaging and PET imaging biomarkers in distinguishing different etiologies of neurodegenerative dementias, including atypical subtype; and (3) discuss the utility of MR imaging and PET abnormalities in predicting future cognitive decline and disease progression individually and in combination.
\end{abstract}

Financial Disclosure: Dr. Wolk is a consultant/advisor to GE Healthcare. Drs. Wolk and Nasrallah have a scientific study/trial with AVID Radiopharmaceuticals/ Eli Lilly. The authors of this article have indicated no other relevant relationships that could be perceived as a real or apparent conflict of interest.

CME Credit: SNMMI is accredited by the Accreditation Council for Continuing Medical Education (ACCME) to sponsor continuing education for physicians. SNMMI designates each JNM continuing education article for a maximum of 2.0 AMA PRA Category 1 Credits. Physicians should claim only credit commensurate with the extent of their participation in the activity. For CE credit, SAM, and other credit types, participants can access this activity through the SNMMI website (http://www.snmmilearningcenter.org) through December 2017.

Neurodegenerative diseases, such as Alzheimer disease, result in cognitive decline and dementia and are a leading cause of mortality in the growing elderly population. These progressive diseases typically have an insidious onset, with overlapping clinical features early in the disease course that make diagnosis challenging. The neurodegenerative diseases are associated with characteristic, although not completely understood, changes in the brain: abnormal protein deposition, synaptic dysfunction, neuronal injury, and neuronal death. Neuroimaging biomarkers-principally regional atrophy on structural MR imaging, patterns of hypometabolism on ${ }^{18} \mathrm{~F}-\mathrm{FDG}$ PET, and detection of cerebral amyloid plaque on amyloid PET-are able to evaluate the patterns of these abnormalities in the brain to improve early diagnosis and help predict the disease course. These techniques have unique strengths and synergies in multimodality evaluation of the patient with cognitive decline or dementia. This review discusses the key imaging biomarkers from MR imaging, ${ }^{18} \mathrm{~F}-\mathrm{FDG}$ PET, and amyloid PET; the imaging features of the most common neurodegenerative dementias; the role of various neuroimaging studies in differential diagnosis and prognosis; and some promising imaging techniques under development.

Key Words: Alzheimer disease; amyloid PET; FDG PET; MRI; neurodegeneration

J Nucl Med 2014; 55:2003-2011

DOI: 10.2967/jnumed.114.141416

$\mathbf{N}$

eurodegenerative diseases cause progressive cognitive decline and ultimately dementia and, with the increasing size of the elderly population, are affecting a growing number of people. Alzheimer disease (AD) is the leading cause of neurodegenerative dementia, followed by dementia with Lewy bodies (DLB), frontotemporal lobar degeneration (FTLD), and even the rarer syndromes

Received Sep. 22, 2014; revision accepted Nov. 4, 2014

For correspondence or reprints contact: Ilya M Nasrallah, 3400 Spruce St.,

Hospital of the University of Pennsylvania, Philadelphia, PA 19104.

E-mail: ilya.nasrallah@uphs.upenn.edu

Published online Nov. 20, 2014.

COPYRIGHT (C 2014 by the Society of Nuclear Medicine and Molecular Imaging, Inc. of progressive supranuclear palsy and corticobasal degeneration. These syndromes are caused by progressive neuronal dysfunction and loss, which result in characteristic symptoms and features in the mid to late course of the disease; however, overlap in cognitive and behavioral profiles can make them difficult to distinguish clinically at presentation. This review introduces clinical aspects of neurodegenerative dementia, provides an overview of imaging modalities, reviews the imaging features of specific neurodegenerative dementias, discusses the use of imaging biomarkers for differential diagnosis and prognosis, and touches on future directions for imaging in neurodegenerative dementia. Selected appropriately, multimodality imaging with MR imaging and PET has the potential to improve diagnosis and management of patients with neurodegenerative dementia.

Although no definitive disease-modifying therapies are yet available, appropriate and early diagnosis allows selection of those interventions and symptomatic treatments most likely to provide benefit, as well as avoidance of therapies that are not likely to help but may cause side effects. Furthermore, early diagnosis allows patients to make plans and prepare for caretakers before cognitive capacity is lost. Although current therapies offer modest symptomatic benefit and may delay institutionalization, there are considerable ongoing efforts toward novel treatments; such therapies would likely be most efficacious if started early in the disease course before significant neurodegeneration occurs. The current benefits of early diagnosis are modest and difficult to quantify, leaving controversy over whether early screening should be performed (1).

Even in affluent countries, it is estimated that at least half of dementia cases are undiagnosed (2). Diagnosis of neurodegenerative dementia is even more challenging at earlier stages, when symptoms are subtle and the characteristic syndromic features may not yet have completely manifested. The initial cognitive effects of neurodegeneration may be masked by compensatory mechanisms (termed cognitive reserve, which varies among individuals depending on factors such as education) and by patient comorbidities such as coexisting depression. Additionally, there is heterogeneity in the clinical phenotype of the neurodegenerative condition that may obfuscate diagnosis. Most prominently, AD is associated with several atypical presentations that are relatively common, particularly in early-onset dementia (before age $65 \mathrm{y}$ ). 
For example, a primary progressive aphasia (PPA) variant of AD presents with language difficulties instead of the more typical amnesia (3) and, thus, is often confused with PPA due to FTLD spectrum neurodegeneration.

Neuroimaging biomarkers may assist in the diagnosis of neurodegenerative dementia and may provide prognostic information. Structural MR imaging, ${ }^{18}$ F-FDG PET, and, more recently, amyloid PET may be useful adjuncts to clinical examination; novel MR imaging techniques and new PET radiotracers under development may further expand our diagnostic ability. These and other biomarkers of dementia can be classified into 2 groups: those that evaluate an underlying molecular pathologic condition, such as amyloid PET, and those that evaluate for evidence of neurodegeneration, including structural MR imaging and ${ }^{18}$ F-FDG PET. Perfusion SPECT is also used to evaluate for regional perfusion abnormalities; patterns tend to match those for ${ }^{18} \mathrm{~F}$-FDG PET, but SPECT has technical disadvantages and poorer accuracy than ${ }^{18} \mathrm{~F}-\mathrm{FDG}$ PET (4) and is not discussed further in this review. As is the case for all diseases of the elderly, evaluation of biomarkers must be considered in the setting of the normal-volume, neural, and synaptic losses of aging. This parallels considerations for standard psychometric testing also used for evaluation of cognitive decline, the results of which depend on language, education, and culture. Comparison to age- and sex-matched normative standards are important for the imaging specialist visually interpreting these studies, particularly when quantifying data from imaging tests (5).

Structural MR imaging is a mainstay of evaluation of patients with cognitive deficits to help exclude nonneurodegenerative etiologies such as vascular disease. Further, structural MR imaging can show evidence of focal atrophy to suggest specific neurodegenerative diseases. Although some atrophy patterns are clear in advanced stages, quantitative postprocessing techniques may assist in early diagnosis of neurodegenerative dementias based on regional gray matter volume and thickness. Limitations of MR imaging include contraindications in patients with pacemakers and other implanted devices and degradation of imaging findings due to patient motion.

PET is used to evaluate a growing number of molecular targets in the brain. Clinical PET, however, is limited to the evaluation of cerebral metabolism using ${ }^{18} \mathrm{~F}-\mathrm{FDG}$ and newer amyloid imaging agents. The distribution of ${ }^{18} \mathrm{~F}-\mathrm{FDG}$ in the brain depends on regional metabolism and blood flow, which change in response to synaptic activity and cell density. Both of these are affected in neurodegenerative dementias, with regional differences allowing for discrimination of the underlying etiology. Sensitivity to synaptic activity can also be a limitation of ${ }^{18} \mathrm{~F}$-FDG PET, as centrally active medications and comorbid psychiatric illness, such as depression, can change or obscure patterns from underlying neurodegeneration (6).

Amyloid PET evaluates for the presence of fibrillar $\beta$-amyloid deposits, one of the hallmark pathologic substrates of AD. Three amyloid radiotracers are approved by the U.S. Food and Drug Administration for clinical use: ${ }^{18} \mathrm{~F}$-florbetaben, ${ }^{18} \mathrm{~F}$-florbetapir, and ${ }^{18} \mathrm{~F}$-flutemetamol, although most studies of amyloid PET have used the first agent developed, ${ }^{11} \mathrm{C}$-Pittsburgh compound B (7). Although there are some differences, these agents appear to perform similarly well for the detection of cerebral amyloid (8). Amyloid PET shows detectable cortical uptake with high sensitivity and specificity when a moderate to severe burden of plaque is present (9). Clinically, amyloid PET is interpreted dichotomously as positive, indicating the presence of cortical amyloid, or negative. Limitations of amyloid PET include prominent nonspecific white matter uptake for clinically available radiotracers, making visual evaluation more difficult. Furthermore, cortical amyloid is not specific for the presence of cognitive symptoms, affecting positive predictive value. Beyond amyloid, there are numerous other pathologic proteins of interest for neurodegenerative diseases, including tau and $\alpha$ synuclein, which are targets for tracer development (10).

Medical images have traditionally been interpreted visually by imaging specialists and referring clinicians. To better quantify these data, several visual rating scales have been developed. However, many of the changes seen in neurodegeneration are difficult or impossible to evaluate by the human eye, and high interrater reliability can be difficult to achieve with visual rating scales. Much research has used manual delineation of regions of interest or semi- or fully automated computational tools for data extraction and quantification, such as voxel-based morphometry. These methods allow for global and regional quantification of brain volume or cortical thickness for MR imaging and of radiotracer distribution and kinetics for PET. Computational methods can generate and evaluate numerous regions of interest, and some groups have attempted to reduce this complexity to summative scores $(11,12)$. Many studies have shown superiority for more quantitative approaches $(13,14)$. Although powerful, many computational approaches are not fully standardized and can be difficult to implement, let alone validate, at other institutions.

\section{NORMAL AGING}

Loss of cerebral volume is typical during normal aging. These changes include global cerebral as well as more regional volume loss (Fig. 1), particularly involving the prefrontal cortex (15). Hippocampal atrophy rates are also higher than global atrophy rates in cognitively normal individuals and increase with age $(15,16)$. Normally, gray matter shows high uptake of glucose; there are mild regional decreases with aging (11) that do not match typical patterns associated with neurodegeneration. Amyloid PET has high sensitivity for cerebral amyloid plaque and is often negative in cognitively normal individuals. However, it is well established that cognitively normal individuals can have detectable cerebral amyloid deposition. The prevalence of cerebral amyloid increases from $10 \%-15 \%$ at age 65 y to about $50 \%$ at age $85 \mathrm{y}$ (17). This uptake in cognitively normal individuals is thought to reflect preclinical AD (18).

\section{AD}

$\mathrm{AD}$ is usually characterized primarily by memory dysfunction with an insidious onset and a long preclinical phase. Although pathologic evaluation for the presence of $\beta$-amyloid neuritic plaques and tau protein-containing neurofibrillary tangles is required for a definitive diagnosis, recent diagnostic criteria have identified biomarkers that can increase confidence in the diagnosis of underlying AD (19). Interest in early diagnosis has identified 2 predementia groups. The prodromal stage is clinically synonymous with mild cognitive impairment (MCI), defined by the presence of objective cognitive impairment without significant functional decline (20). However, patients in the prodromal stage are a heterogeneous group, and many MCI patients—sometimes the majority, depending on the method of classification-do not have underlying $\mathrm{AD}$. Recent criteria have used the presence of biomarker evidence of amyloid disease and neurodegeneration to increase confidence that MCI represents prodromal AD (20). Patients in 


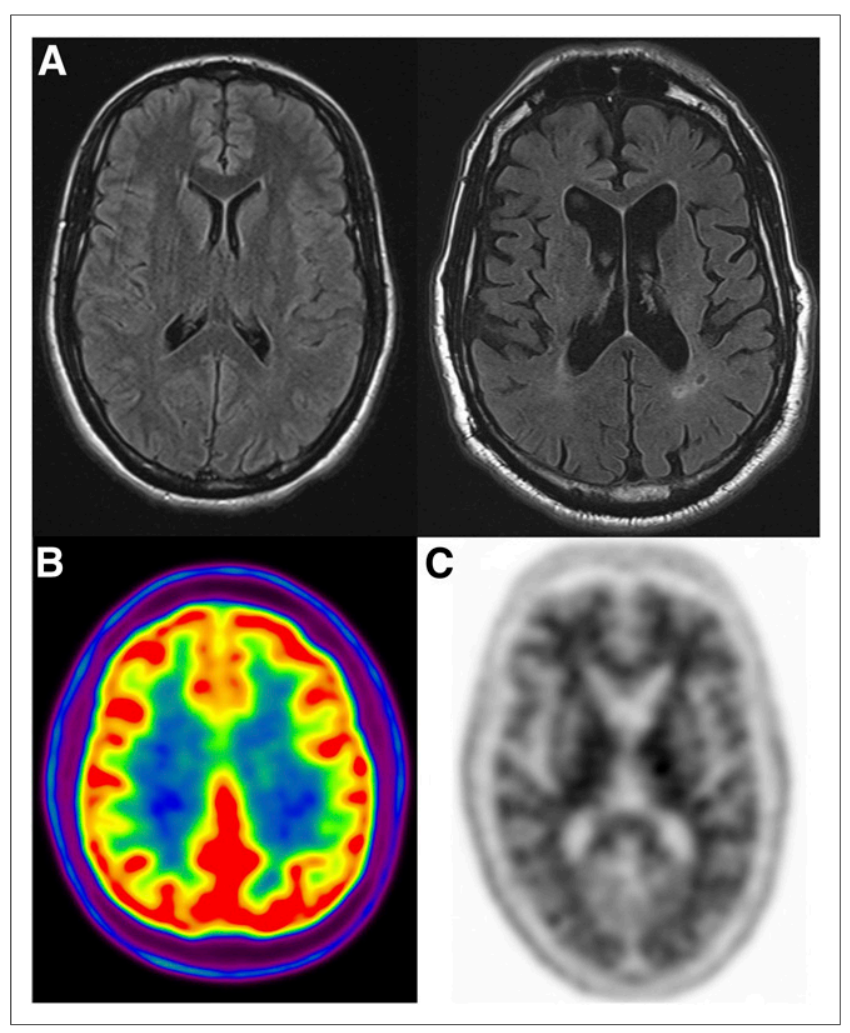

FIGURE 1. Imaging of cognitively normal individuals. (A) Axial fluidattenuated inversion recovery MR imaging of brain shows typical appearance in adult in third decade of life (left; normal findings) and adult in eighth decade of life (right; mild atrophy and mild white matter hyperintensities). (B) Normal ${ }^{18}$ F-FDG PET findings in a third individual show high uptake in gray matter structures. (C) Normal ${ }^{18} \mathrm{~F}$-florbetapir amyloid PET findings in a fourth individual show typical white matter uptake but no evidence of elevated cortical binding.

the preclinical stage of $\mathrm{AD}$ have been defined as those with no objective cognitive deficit or biomarker evidence of neurodegeneration but with evidence of cerebral amyloid deposition (21); these criteria were developed for research purposes, with the hope that defining this group could lead to future preventative or diseasemodifying treatments at this stage.

The hallmark MR imaging biomarker for $\mathrm{AD}$, and by far the most studied in the entire field of neurodegeneration, is hippocampal atrophy (Fig. 2). The average hippocampal volume reduction is $20 \%-$ $25 \%$ in $\mathrm{AD}$ and $10 \%-15 \%$ in MCI (22). Visual assessment for pathologic hippocampal atrophy at early stages is challenging, particularly in MCI, resulting in lower sensitivity for AD. Automated methods for hippocampal evaluation have been developed but are not widely available (23). The lack of a standardized methodology for measurement of hippocampal volumes has limited the incorporation of this biomarker into clinical practice. Computational evaluation of the entire brain for evidence of focal-volume or cortical-thickness reductions shows other characteristic regions affected in $\mathrm{AD}$, such as the precuneus and lateral parietal lobes; these $\mathrm{AD}$ signature patterns correlate with the severity of cognitive decline $(12,24)$.

${ }^{18}$ F-FDG PET shows characteristic hypometabolism in the hippocampi or medial temporal lobes, posterior cingulate, precuneus, and lateral temporoparietal cortex, with the most reliable early changes seen in the posterior cingulate cortex (Fig. 2) $(25,26)$. Milder but similar regional changes are seen in cases of MCI that are likely to progress to clinical AD (5), whereas more advanced
AD typically shows frontal lobe involvement. It has long been held that metabolism may become abnormal earlier in the course of $\mathrm{AD}$ than MR imaging is capable of measuring (27), but some recent evidence suggests that these neurodegenerative biomarkers may change simultaneously (28). In typical amnestic AD, hypometabolism is usually bilateral but may be asymmetric (29) and spares the basal ganglia, somatosensory cortex, and occipital cortex (26,30). ${ }^{18}$ F-FDG PET can discriminate AD patients from healthy controls with high sensitivity and specificity (11). Use of ${ }^{18} \mathrm{~F}$-FDG PET has been shown to improve accuracy in diagnosis of AD compared with clinical evaluation (31) and to change diagnosis in about a quarter of patients (32). Amyloid PET was positive with high sensitivity for $\mathrm{AD}$ in a series with pathologic confirmation (33). Approximately 50\% of MCI patients show positive amyloid scans, particularly those with deficits in multiple cognitive domains (34). Cerebral amyloid accumulation appears to start decades before the onset of dementia (35) and may be the earliest biomarker to show measurable abnormality (28).

There are several uncommon, atypical presentations of AD: the logopenic variant of PPA, marked by a primary impairment in language; posterior cortical atrophy, primarily associated with

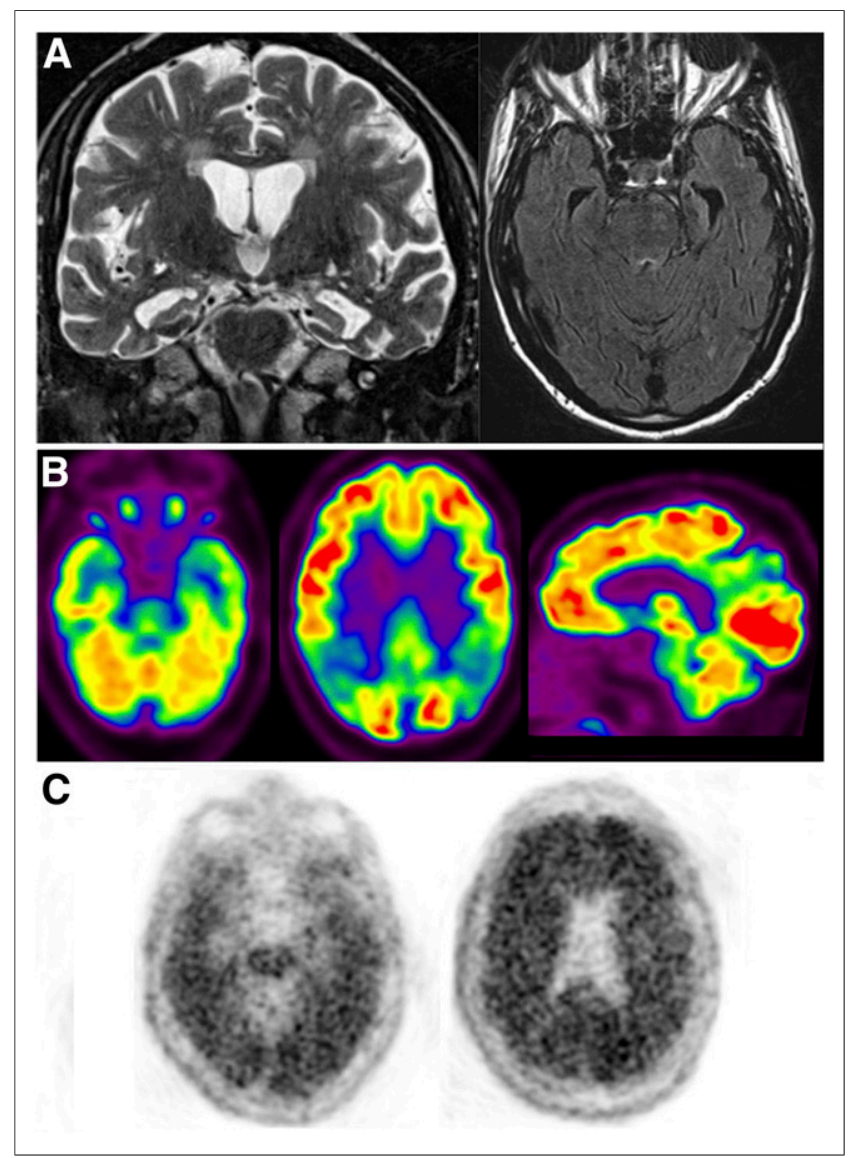

FIGURE 2. Neuroimaging in AD. (A) Coronal T2-weighted and axial fluid-attenuated inversion recovery MR images show marked medial temporal lobe atrophy involving hippocampus and subjacent entorhinal cortex, out of proportion to global volume loss. (B) Axial and sagittal images from ${ }^{18} \mathrm{~F}-\mathrm{FDG}$ PET show typical lateral temporoparietal, posterior cingulate, and medial temporal lobe hypometabolism. Milder frontal hypometabolism is present, also common. (C) Axial images from positive ${ }^{18} \mathrm{~F}$-florbetapir amyloid PET scan show diffuse cortical activity with loss of distinction between gray and white matter. 
visuospatial impairment; and the frontal variant of $\mathrm{AD}$, characterized by changes in behavioral and executive function $(36,37)$. These atypical forms represent about one third of young-onset AD cases and at least $5 \%$ of late-onset $\mathrm{AD}$ cases and pose diagnostic challenges (36). Neuroimaging demonstrates a distinct anatomic distribution of gray matter atrophy and hypometabolism consistent with the clinical presentation of these conditions. The logopenic variant of PPA tends to display greater left perisylvian or temporoparietal decreases, and posterior cortical atrophy is associated with parietal-occipital decreases (38). The pattern of amyloid deposition seen at autopsy and on amyloid PET in these atypical presentations is not clearly distinct from typical AD (39).

\section{FTLD}

FTLD is a heterogeneous group of neurodegenerative conditions that share clinical features and pathologic and genetic etiologies. The behavioral variant is the most common subtype, clinically characterized by early personality and behavior changes. Semantic dementia and progressive nonfluent aphasia demonstrate primary language changes. There are also overlap syndromes with mixed features. Age at onset is typically younger than for $\mathrm{AD}$, generally before $65 \mathrm{y}$. FTLD can be associated with abnormal aggregates of various proteins, including primarily tau-based disease, transactive response DNA binding protein 43 , or fused in sarcoma (3).

MR imaging demonstrates frontal and anterior temporal atrophy, which can be severe, resulting in a knife-blade-gyri appearance. Subtypes of FTLD differ in atrophy patterns (Fig. 3). The behavioral variant of FTLD shows the greatest atrophy in the anterior frontal lobes, semantic dementia typically involves the anterior temporal lobes, and progressive nonfluent aphasia typically involves the left anterior perisylvian region, particularly the left ventrolateral prefrontal cortex. Asymmetry is common, particularly in language variants, in which the left side is more severely affected (40). Regional atrophy patterns have been used to distinguish these 3 sub-

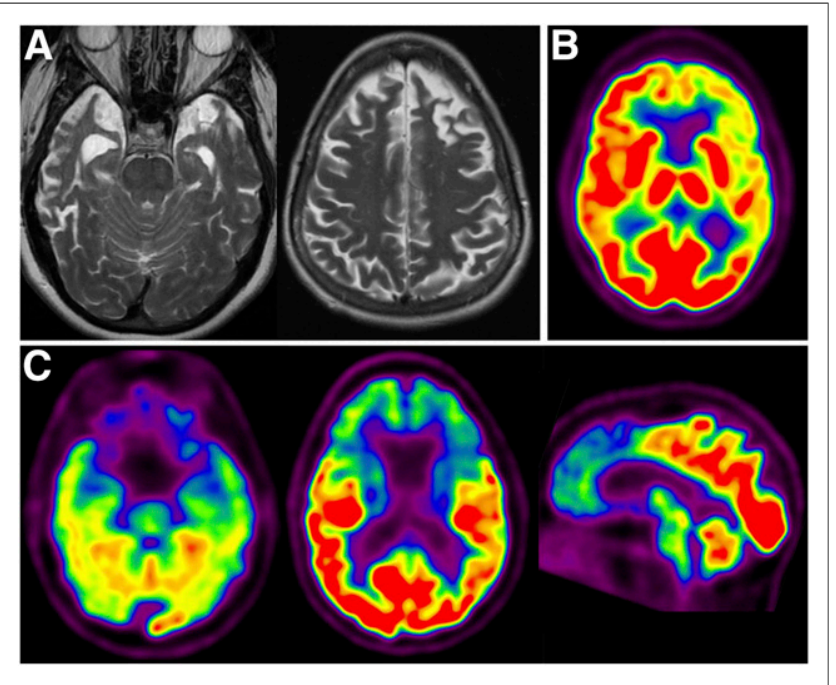

FIGURE 3. Neuroimaging in FTLD. (A) Axial T2-weighted images from 2 patients show 2 characteristic patterns of FTLD: anterior temporal (left) and frontal (right) atrophy. Both show asymmetry and knife-blade appearance of gyri. (B and C) ${ }^{18} \mathrm{~F}-\mathrm{FDG}$ PET shows 2 typical patterns: left frontotemporoparietal $(\mathrm{B}$; axial) and frontal-predominant $(\mathrm{C}$; axial and sagittal) hypometabolism. types of FTLD with high sensitivity and specificity (41). There is some evidence that atrophy patterns may relate to underlying pathologic subtype (42). However, this link is probabilistic at best: on an individual level, each atrophy pattern can be seen in the setting of multiple clinical subtypes of FTLD-spectrum diseases. There are corresponding patterns seen on ${ }^{18}$ F-FDG PET (Fig. 3). The behavioral variant of FTLD shows predominately frontal hypometabolism, with varying involvement of the anterior cingulate cortex and anterior temporal lobes (43). Semantic dementia is associated with predominately anterior temporal lobe hypometabolism that is usually asymmetric (44), whereas progressive nonfluent aphasia shows predominantly asymmetric left anterior perisylvian hypometabolism (45). Rarely, FTLD can present with an AD pattern of hypometabolism, although usually associated with frontal hypometabolism (5). Additionally, amyloid PET is expected to be negative in FTLD, but a significant minority of patients have positive scans, with rates differing depending on FTLD subtype; it is thought that these cases represent comorbid AD or, more likely, misdiagnosis of the underlying etiology $(46,47)$.

There are several less common neurodegenerative etiologies of dementia, including progressive supranuclear palsy and corticobasal degeneration, which have been classified both as within the FTLD spectrum and as atypical Parkinsonian syndromes, given their association with characteristic motor symptoms. Progressive supranuclear palsy is characterized by oculomotor abnormality and gait instability, with less frequent executive-function, visuospatial, and language impairments. MR imaging shows profound midbrain atrophy resulting in what has been termed the hummingbird appearance of the brain stem as well as pontine, thalamic, striatal, and milder frontal cortical atrophy. Superior cerebellar peduncle atrophy is characteristic (Fig. 4). ${ }^{18} \mathrm{~F}-\mathrm{FDG}$ PET may show symmetric prefrontal and primary or supplemental motor cortex hypometabolism, also with deep nuclear and midbrain hypometabolism (30). Corticobasal degeneration is often associated with a clinical constellation of findings termed corticobasal syndrome, which includes asymmetric parkinsonism, apraxia, gait disturbance, and alien hand phenomenon. In corticobasal degeneration, there is asymmetric frontoparietal atrophy involving paracentral and parasagittal structures (Fig. 4) (48). ${ }^{18}$ F-FDG PET shows asymmetric, often unilateral, hypometabolism contralateral to predominant motor symptoms, involving the parietotemporal cortex, sensorimotor cortex, prefrontal cortex, caudate, and thalamus; asymmetry and sensorimotor cortex involvement are relatively unique (30). Corticobasal syndrome lacks specificity with regard to the underlying disease, as it can be seen with other FTLD-associated diseases, as well as AD. Conversely, typical corticobasal degeneration can present as syndromes similar to progressive supranuclear palsy and progressive nonfluent aphasia, among others (49).

\section{DLB}

DLB is the second most common form of neurodegenerative dementia. Pathologically similar to Parkinson disease, with $\alpha$-synuclein-containing Lewy bodies seen on pathologic examination, DLB is clinically distinguished from Parkinson disease dementia by presentation of dementia before or within $1 \mathrm{y}$ of the onset of parkinsonian movement symptoms (50). At least $70 \%-80 \%$ of DLB patients have cerebral amyloid plaque (51), with many cases meeting the pathologic criteria for mixed DLB-AD. The core clinical features of DLB are parkinsonism, cognitive fluctuations, and visual hallucinations. Cognitive and behavior features include 


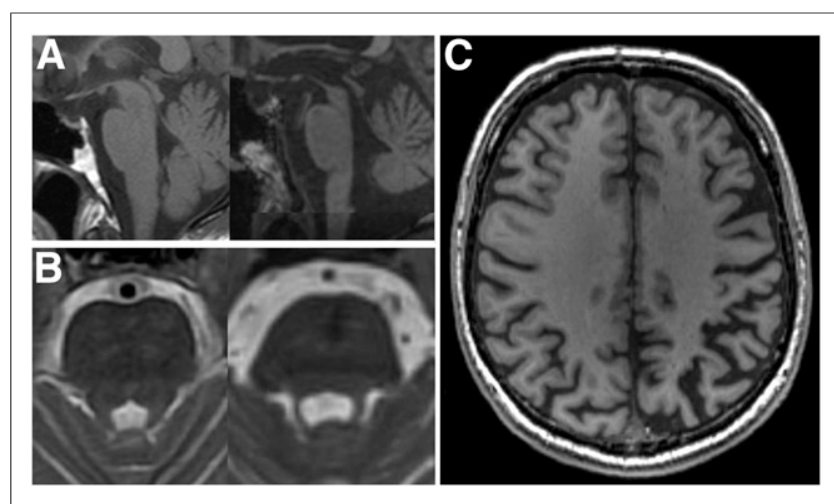

FIGURE 4. MR imaging appearance of progressive supranuclear palsy and corticobasal degeneration subtypes of FTLD. Normal sagittal T1weighted and axial T2-weighted images centered on brain stem are shown for comparison on left in parts $A$ and $B$. (A) Patient with progressive supranuclear palsy showing severe midbrain atrophy with hummingbird appearance of brain stem, characterized by shortened anteroposterior dimension of midbrain, and concave superior margin. (B) Patient with progressive supranuclear palsy showing superior cerebellar peduncle atrophy. (C) Axial T1-weighted image of patient with corticobasal degeneration showing asymmetric left frontoparietal atrophy.

abnormalities of executive function, attention, and visuospatial ability; hallucinations; depression; and anxiety (50).

Structural MR imaging results have been variable, with cortical atrophy probably being due to associated $\mathrm{AD}$, as studies show that subjects with DLB but no AD have brain volumes similar to those of healthy controls, including the volumes of the medial temporal structures (52). ${ }^{18}$ F-FDG PET shows occipital hypometabolism in addition to involvement of regions affected by $\mathrm{AD}$ : the posterior cingulate cortex, temporoparietal cortex, and, less prominently, frontal cortex, although medial temporal metabolism is generally preserved (Fig. 5) (30,53). Greater hypometabolism is correlated with more severe dementia (53). Amyloid PET is usually positive in DLB but usually negative in Parkinson disease dementia (51). Unlike other neurodegenerative dementias, DLB shows abnormally decreased tracer uptake in the striatum on PET and SPECT imaging of the dopaminergic system, with ${ }^{123}$ I-FP-ioflupane (GE Healthcare) being the most widely used tracer (54).

\section{DIFFERENTIAL DIAGNOSIS OF DEMENTIA}

In addition to a sometimes overlapping clinical presentation, various etiologies of neurodegenerative dementia can have a similar appearance on a variety of imaging modalities. Most imaging studies have focused on distinction from healthy control groups; however, differential diagnosis has also been investigated, with studies generally showing improvement in diagnosis compared with clinical evaluation. Although studies showing high accuracy using biomarkers to differentiate between typical patients and healthy controls demonstrate important features of disease, the studies most relevant to clinical evaluation will interrogate the performance of the biomarkers earlier in the disease course and in less typical presentations for which clinical assessment has poorer performance; these studies are, however, difficult to perform, largely because of the challenge of confirming the true diagnosis. Thus, although many measures have shown a strong ability to distinguish between groups in controlled studies, the utility in evaluation of individual patients will be lower because of overlaps in biomarker results in these more challenging cases.
MR imaging has a central role in the differential diagnosis of dementia. It is primarily used to exclude nonneurodegenerative etiologies of dementia (Fig. 6). Vascular dementia, or vascular cognitive impairment, is arguably the second most common etiology of dementia and is typically associated with severe small-vessel ischemic changes and lacunar infarcts, although patients with largeterritory infarcts can also present with dementia. Normal-pressure hydrocephalus may be suggested by ventricular enlargement, although imaging is far from definitive in this population. Sporadic Creutzfeldt-Jakob disease, a prion disease, is often characterized by cortical, basal ganglionic, and medial thalamic diffusion restriction, frequently with associated fluid-attenuated inversion recovery abnormalities. Other conditions, including paraneoplastic, infectious, and inflammatory conditions, can cause cognitive decline and may be identified on MR imaging. Beyond excluding alternative etiologies, MR imaging has utility in distinguishing the etiologies of neurodegenerative disease: studies using automated image analysis software have found that MR imaging can be used to distinguish AD from FTLD (55), as well as distinguishing subtypes of FTLD (56). Preservation of hippocampal and lateral temporoparietal volumes favors DLB over AD (52).

Multiple studies have shown that ${ }^{18} \mathrm{~F}$-FDG PET is useful in the differential diagnosis of dementia, although few studies have compared performance in more than 2 neurodegenerative conditions and most have used clinical diagnosis as the reference standard, limiting generalizability. Normal ${ }^{18}$ F-FDG uptake significantly reduces the likelihood of an underlying neurodegenerative process (31). ${ }^{18}$ F-FDG PET shows high sensitivity and specificity for distinguishing AD from FTLD (13). The presence of occipital hypometabolism can distinguish DLB from AD, which are otherwise similar, with $85 \%-90 \%$ sensitivity and $80 \%-90 \%$ specificity (57). Vascular dementia can also be differentiated from AD by ${ }^{18} \mathrm{~F}-\mathrm{FDG}$ PET, showing focal asymmetric hypometabolism with more common involvement of the deep nuclei (58). The few studies that have included mixed cohorts have shown high performance in differential diagnosis (59), with an estimated $87 \%$ sensitivity (60) and $81 \%$ specificity for distinguishing $\mathrm{AD}$ from other causes of dementia (6). Panegyres et al. (6) showed $78 \%$ sensitivity and $81 \%$ specificity for detection of AD versus other conditions included in the study: FTLD, DLB, PPA, and depression. Specificity was more than $95 \%$ for diagnosis of the other etiologies; however, sample sizes were small. Topography on PET generally shows a strong correlation with the clinical syndrome but may be less accurate in distinguishing the underlying pathologic condition, such as in some subtypes of PPA in which several different conditions

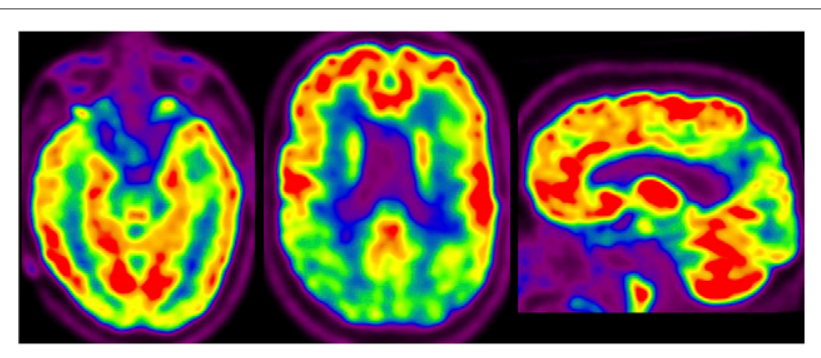

FIGURE 5. ${ }^{18} \mathrm{~F}-\mathrm{FDG}$ PET in DLB. Axial images through levels of temporal lobes and lateral ventricles (left and middle) and parasagittal image (right) show occipital and posterior temporoparietal hypometabolism, with sparing of posterior cingulate cortex and medial temporal lobes. 


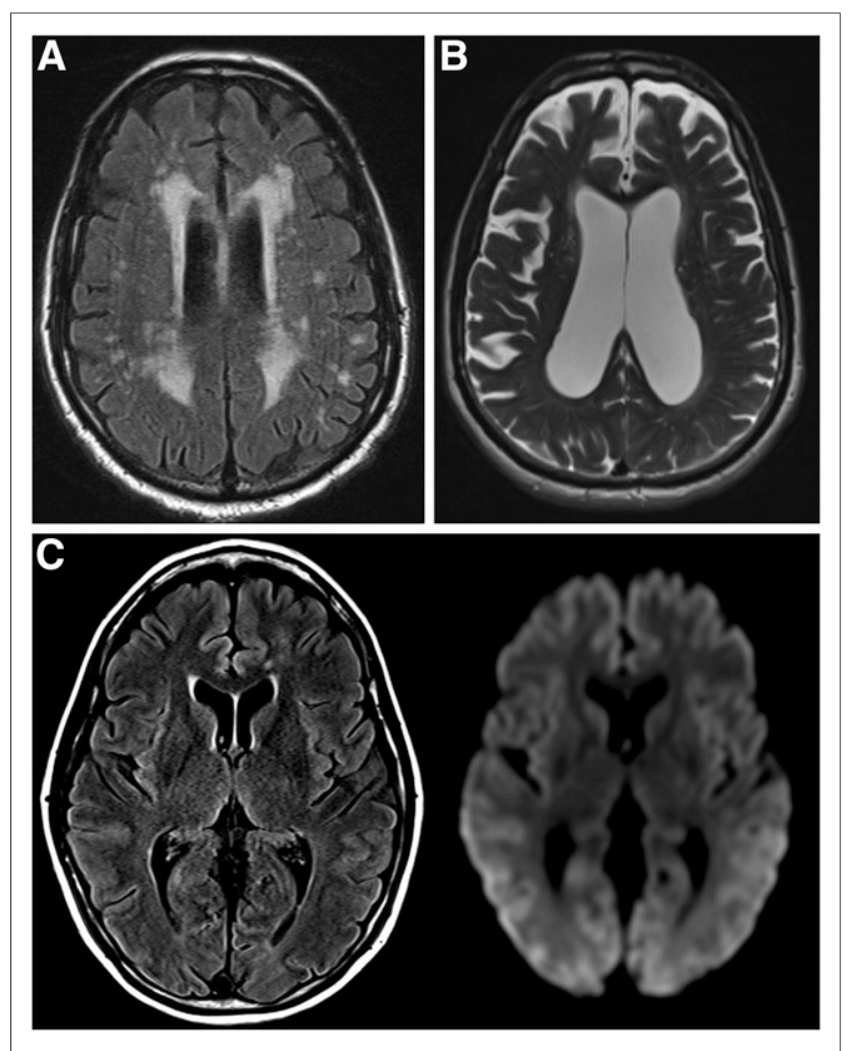

FIGURE 6. MR imaging appearance of selected secondary causes of dementia. (A) Marked small-vessel ischemic changes are manifested by confluent periventricular hyperintensity and subcortical lesions on axial fluid-attenuated inversion recovery image. (B) Axial T2-weighted image shows enlarged ventricles in patient with normal-pressure hydrocephalus, out of proportion to degree of diffuse atrophy. (C) Axial fluid-attenuated inversion recovery and diffusion-weighted images in patient with CreutzfeldtJakob disease show diffuse cortical and asymmetric deep gray matter signal abnormality, most evident on diffusion imaging.

can have similar clinical presentations and patterns of neurodegeneration (61). Multiple studies have shown improved accuracy, improved diagnostic confidence, and reduced interrater variability compared with clinical evaluation of neurodegenerative dementia alone $(13,31)$.

Amyloid PET has proven utility in the differential diagnosis of neurodegenerative dementia, being able to identify patients likely to have AD or DLB versus other causes of dementia (46). This utility is highest in cases of early-onset dementia, for which AD and FTLD each accounts for almost half of cases. Amyloid PET and ${ }^{18}$ F-FDG PET have similar accuracy in distinguishing these diagnoses, with amyloid PET showing higher sensitivity (47). Amyloid PET can also be used to distinguish nonamnestic presentations of AD from FTLD and to distinguish amnestic presentations of FTLD from typical AD. PPA, for example, can be associated with either AD or FTLD. Amyloid PET may also be useful for evaluating whether $\mathrm{AD}$ is present in patients with neurologic and psychiatric comorbidities, which may both interfere with neurocognitive testing and alter the metabolic pattern of ${ }^{18}$ F-FDG PET but should not affect amyloid PET. Vascular dementia is an important differential diagnosis for AD with distinct therapeutic options; however, patients with severe smallvessel ischemic changes of the white matter or multiple infarcts evident on MR imaging may have atypical findings on MR im- aging or ${ }^{18}$ F-FDG PET. Amyloid PET is not useful in distinguishing $\mathrm{AD}$ from DLB (46).

\section{IMAGING BIOMARKERS FOR PROGNOSIS}

Biomarkers have been extensively evaluated for efficacy in determining the progression of cognitive decline. These include neurocognitive test results, genetic tests, vascular risk factors, and cerebrospinal fluid sampling, in addition to imaging biomarkers (62); this review focuses on imaging biomarkers. There are several limitations in the application of biomarker studies to clinical use. First, the fact that the patient population included in a research study is likely to differ from that of any particular clinical setting can have an effect on biomarker utility (63). Proven utility of a biomarker in one phase of disease does not indicate that it would be able to inform on prognosis at another stage, as biomarkers appear to have a characteristic dynamic period (28). Furthermore, although many studies derive methods for biomarker use, these need to be validated and standardized in independent, larger samples.

Numerous studies have evaluated associations between brain structural measurements on MR imaging and progression of cognitive decline and onset of dementia. Cross-sectional medial temporal lobe and hippocampal volume and longitudinal measures of rate of atrophy are sensitive and predictive structural features for future decline. Worsening medial temporal lobe atrophy is associated with greater progression to $\mathrm{AD}$ both for cognitively normal individuals and for MCI patients (64). Global atrophy is also a risk factor. Patients with atrophy on MR imaging at the time of diagnosis of MCI have demonstrated greater rates of conversion to AD within 3-5 y than those without atrophy (65). Similarly, progressors to $\mathrm{AD}$ among $\mathrm{MCI}$ patients and cognitively normal individuals have higher rates of ventricular enlargement than do individuals who remain stable $(65,66)$. Unfortunately, hippocampal atrophy is not specific to $\mathrm{AD}$; for example, it is frequently observed in some types of FTLD. Associations between atrophy and progression have been shown in other neurodegenerative dementias. FTLD patients with frontal atrophy had greater rates of cognitive decline than temporal variants (67). Atrophy rates in DLB correlated with worsening cognitive decline (68). However, although sensitive in group analyses, there is overlap between groups for structural MR imaging measures, including hippocampal volume in $\mathrm{AD}$, which may limit usefulness for prognosis in individual patients (65).

${ }^{18} \mathrm{~F}$-FDG PET has also been shown to provide important prognostic information for progression of cognitive decline. ${ }^{18} \mathrm{~F}-\mathrm{FDG}$ PET shows high specificity; negative ${ }^{18}$ F-FDG PET indicates that progression from MCI to dementia is unlikely $(5,6)$. Hypometabolism in $\mathrm{AD}$-characteristic regions including the posterior cingulate cortex, lateral temporoparietal lobes, and medial temporal lobes is predictive of conversion from MCI to dementia (29). Declining hypometabolism in MCI and $\mathrm{AD}$ is associated with greater cognitive and functional decline (29). Use of ${ }^{18}$ F-FDG PET for prognosis of other forms of neurodegenerative dementia has been studied much less. In one small study, nondemented individuals who showed occipital hypometabolism were at increased risk for conversion to DLB, especially if temporoparietal hypometabolism was also present (69). ${ }^{18} \mathrm{~F}$-FDG PET also appears to predict neurodegeneration before symptom onset in carriers of a mutation associated with FTLD (70).

The presence of cerebral amyloid deposition has prognostic information for cognitively normal individuals and MCI patients. 
Most studies of cerebral amyloid have shown relative stability in demented patients, suggesting that changes in amyloid burden are not likely to provide clinically useful information at this stage in the absence of a treatment intervention that modulates amyloid deposition (26). Cerebral amyloid can be detected years, even decades, before the onset of cognitive decline (35). Cognitively intact patients with positive amyloid PET findings have been reported by some, but not all, studies as displaying poorer performance on cognitive testing (71). There is also an association between amyloid positivity and higher hippocampal atrophy rates in healthy and MCI individuals (16). Finally, MCI and cognitively normal individuals with amyloid have higher rates of conversion to $\mathrm{AD}$ and $\mathrm{MCI}$, respectively $(35,72)$. However, it remains uncertain whether all cognitively normal individuals with a positive amyloid scan will progress to dementia. The predictive value with regard to the timing of cognitive decline is also unclear, with many individuals still not displaying decline more than $5 \mathrm{y}$ after a positive study (73). Nonetheless, the study of amyloid-positive, cognitively normal individuals is a major focus of research in the field.

Fewer studies have compared the relative efficacy and contribution of different biomarkers for estimation of prognosis in neurodegenerative dementia. Imaging biomarkers in many cases show improved predictive ability compared with clinical evaluation or neurocognitive testing (74), although this finding is not uniform. Several studies have shown a slightly higher predictive ability for ${ }^{18} \mathrm{~F}$-FDG PET than for structural MR imaging in separating MCI from cognitively normal controls and in determining progression from MCI to AD (74), with a smaller number of studies showing similar performance (75). Rowe et al. have shown that amyloid PET positivity is a stronger predictor than hippocampal volume that cognitively normal individuals will progress to $\mathrm{MCI}$ and that MCI will progress to $\mathrm{AD}$, with a high positive predictive value (72). One study comparing amyloid PET and ${ }^{18} \mathrm{~F}$ FDG PET for conversion of MCI to AD showed higher sensitivity for amyloid PET, higher specificity for ${ }^{18} \mathrm{~F}$-FDG PET, and similar accuracy between the two (14). Prestia et al. evaluated multiple biomarkers in 2 different cohorts and showed that amyloid measures had the highest sensitivity for progression to AD and hippocampal volumes the highest specificity to exclude stable MCI, with ${ }^{18}$ F-FDG PET showing intermediate values for both (63).

Combining biomarker data can improve prognostic ability. Adding cognitive score results to measures of brain atrophy improved the ability to predict future conversion from MCI to AD (76). The combination of regional volumes and CSF biomarkers showed a modestly improved ability to predict conversion of MCI to $\mathrm{AD}$ over the use of either alone, with a combined accuracy of $69 \%$ (77). The combination of CSF measurements of tau protein and hippocampal atrophy on MR imaging was shown to identify-from a population of cognitively intact individuals - those who are likely to show deterioration in cognitive testing (78). Classification schemes using baseline MR imaging, PET, and CSF biomarkers have shown improved accuracy in distinguishing $\mathrm{MCI}$ patients from cognitively normal individuals and in predicting conversion from MCI to $\mathrm{AD}(74,75)$. A general principle that appears to be emerging is that amyloid PET may provide a more certain etiologic diagnosis but that neurodegenerative biomarkers, such as ${ }^{18}$ F-FDG PET and structural imaging, may better track disease severity and the timing of progression in MCI patients and perhaps cognitively normal individuals (35). Thus, these methods serve complementary roles.

\section{FUTURE IMAGING BIOMARKERS AND TECHNOLOGY}

Novel MR imaging sequences and PET radiotracers have the potential to further improve evaluation of patients with cognitive decline and dementia. These novel MR imaging sequences and radiotracers are being applied in research settings and have been informative on the pathophysiology of neurodegenerative dementia but not yet adequately validated for routine clinical use.

Perhaps the most likely MR imaging sequence to translate to the clinic is arterial spin labeling, which measures cerebral perfusion. Arterial spin labeling shows hypoperfusion in regions similar to those seen on ${ }^{18} \mathrm{~F}$-FDG PET and with SPECT perfusion agents in AD (79). Furthermore, differences in cerebral perfusion between $\mathrm{AD}$ and FTLD on arterial spin labeling MR imaging show promise in differential diagnosis (80). Thus, arterial spin labeling could add information previously evaluated only on PET and SPECT to a routine MR imaging study, although further standardization of this sequence, particularly between MR imaging scanner vendors, will be required. MR imaging can also detect changes in regional neural activation patterns using blood oxygen level-dependent functional MR imaging, which measures local changes in blood oxygenation caused by alterations in synaptic activity. Blood oxygen level-dependent functional MR imaging can be used to evaluate brain activity in patients during specific tasks, such as a language task, or while at rest to assess regions with covaried blood oxygen level-dependent signal reflecting brain network functional connectivity. Studies have detected alterations in blood oxygen level-dependent signal in demented patients, including decreased activation in the hippocampi on memory tasks on functional MR imaging (81) and disruptions in functional connectivity on restingstate functional MR imaging (82). These techniques currently appear more suited to research applications than clinical evaluation.

Numerous PET radiotracers have been developed that can be applied to the evaluation of neurodegenerative disease. There is great interest in newly developed radiotracers with the potential to detect the pattern of deposition of tau protein, including ${ }^{18} \mathrm{~F}-\mathrm{AV}$ 1451 (formerly T807) and ${ }^{18} \mathrm{~F}-\mathrm{THK} 523(10,83)$. Tau is the principal component of neurofibrillary tangles, one of the hallmark neuropathologic features of $\mathrm{AD}$, and forms aggregates in multiple other neurodegenerative diseases, including multiple subtypes of FTLD. Tau aggregates are neurotoxic; the presence of tau aggregates is associated with neurodegeneration. The degree and distribution of neurofibrillary tangles are more closely related to the pattern and severity of clinical symptoms in AD than amyloid (84). The ability to provide information on the spatial distribution and extent of tau deposition makes these PET tracers more likely to better track disease severity than a scalar, global measure such as cerebrospinal fluid tau. PET radiotracers targeting neuroinflammation or microglial activation have also shown promise in studying AD in early studies, revealing significant differences between AD and cognitively normal groups and correlation of binding with clinical severity (85).

Recent technologic advancements have allowed construction of combined PET/MR imaging scanners, which have had limited clinical use to date. These combined scanners have the potential to acquire both MR imaging and PET data within the time of a single traditional MR imaging scan, adding diagnostic information without additional time or testing, which can be challenging for patients with dementia. Because postacquisition image coregistration is relatively straightforward for the brain, combining standard MR imaging and PET acquisitions on one system adds little diagnostic 
information. PET/MR imaging scanners are uniquely capable of simultaneously evaluating dynamic processes in the brain, particularly using dynamic PET acquisitions with novel radiotracers and functional MR imaging. These have clear potential for research applications; it remains to be seen whether such information will be clinically useful.

\section{CONCLUSION}

Neuroimaging allows for earlier and more accurate diagnosis and determination of prognosis for neurodegenerative dementia and individuals with cognitive decline. Diagnostic criteria for these conditions are being revised to include imaging biomarker findings, including recent updates for MCI and AD (19). In AD, there is evidence of an ordered sequence of change in imaging biomarkers, with early abnormality on amyloid PET followed by various measures of neurodegeneration quantified on structural MR imaging and ${ }^{18}$ F-FDG PET (28). Increasing efforts to combine biomarker data to further improve diagnostic accuracy must be tested in independent and inclusive samples to prove validity. Selected appropriately, multimodality imaging with MR imaging and PET has the potential to improve diagnosis and management in patients with neurodegenerative dementia, providing complementary information that may be acquired simultaneously with upcoming PET/MR imaging scanners. The value of imaging biomarkers will be greatly enhanced with the development of disease-modifying therapies.

\section{ACKNOWLEDGMENT}

This research was supported by NIH grants AG010124 and AG040271.

\section{REFERENCES}

1. Fox C, Lafortune L, Boustani M, Dening T, Rait G, Brayne C. Screening for dementia: is it a no brainer? Int J Clin Pract. 2013;67:1076-1080.

2. World Alzheimer report 2011: the benefits of early diagnosis and intervention. http://www.alz.co.uk/research/world-report-2011. Accessed November 4, 2014.

3. Josephs KA. Frontotemporal dementia and related disorders: deciphering the enigma. Ann Neurol. 2008;64:4-14.

4. Herholz K. Perfusion SPECT and FDG-PET. Int Psychogeriatrics. 2011;23(suppl 2): S25-S31.

5. Mosconi L, Tsui WH, Herholz K, et al. Multicenter standardized ${ }^{18}$ F-FDG PET diagnosis of mild cognitive impairment, Alzheimer's disease, and other dementias. J Nucl Med. 2008;49:390-398.

6. Panegyres PK, Rogers JM, McCarthy M, Campbell A, Wu J. Fluorodeoxyglucosepositron emission tomography in the differential diagnosis of early-onset dementia: a prospective, community-based study. BMC Neurol. 2009;9:41.

7. Mason NS, Mathis CA, Klunk WE. Positron emission tomography radioligands for in vivo imaging of A $\beta$ plaques. J Labelled Comp Radiopharm. 2013;56:89-95.

8. Landau SM, Breault C, Joshi AD, et al. Amyloid-beta imaging with Pittsburgh compound B and florbetapir: comparing radiotracers and quantification methods. J Nucl Med. 2013;54:70-77.

9. Clark CM, Pontecorvo MJ, Beach TG, et al. Cerebral PET with florbetapir compared with neuropathology at autopsy for detection of neuritic amyloid- $\beta$ plaques: a prospective cohort study. Lancet Neurol. 2012;11:669-678.

10. Chien DT, Bahri S, Szardenings AK, et al. Early clinical PET imaging results with the novel PHF-tau radioligand [F-18]-T807. J Alzheimers Dis 2013;34: 457-468.

11. Herholz K, Salmon E, Perani D, et al. Discrimination between Alzheimer dementia and controls by automated analysis of multicenter FDG PET. Neuroimage. 2002;17:302-316.

12. Davatzikos C, Xu F, An Y, Fan Y, Resnick SM. Longitudinal progression of Alzheimer's-like patterns of atrophy in normal older adults: the SPARE-AD index. Brain. 2009;132:2026-2035.

13. Foster NL, Heidebrink JL, Clark CM, et al. FDG-PET improves accuracy in distinguishing frontotemporal dementia and Alzheimer's disease. Brain. 2007; 130:2616-2635.
14. Rabinovici GD, Rosen HJ, Alkalay A, et al. Amyloid vs FDG-PET in the differential diagnosis of AD and FTLD. Neurology. 2011;77:2034-2042.

15. Fjell AM, Walhovd KB, Fennema-Notestine C, et al. One-year brain atrophy evident in healthy aging. $J$ Neurosci. 2009;29:15223-15231.

16. Nosheny RL, Insel PS, Truran D, et al. Variables associated with hippocampal atrophy rate in normal aging and mild cognitive impairment. Neurobiol Aging. August 1, 2014 [Epub ahead of print].

17. Rowe CC, Ellis KA, Rimajova M, et al. Amyloid imaging results from the Australian Imaging, Biomarkers and Lifestyle (AIBL) study of aging. Neurobiol Aging. 2010;31:1275-1283.

18. Sperling RA, Karlawish J, Johnson KA. Preclinical Alzheimer disease: the challenges ahead. Nat Rev Neurol. 2013;9:54-58.

19. McKhann GM, Knopman DS, Chertkow H, et al. The diagnosis of dementia due to Alzheimer's disease: recommendations from the National Institute on AgingAlzheimer's Association workgroups on diagnostic guidelines for Alzheimer's disease. Alzheimers Dement. 2011;7:263-269.

20. Albert MS, DeKosky ST, Dickson D, et al. The diagnosis of mild cognitive impairment due to Alzheimer's disease: recommendations from the National Institute on Aging-Alzheimer's Association workgroups on diagnostic guidelines for Alzheimer's disease. Alzheimers Dement. 2011;7:270-279.

21. Sperling RA, Aisen PS, Beckett LA, et al. Toward defining the preclinical stages of Alzheimer's disease: recommendations from the National Institute on AgingAlzheimer's Association workgroups on diagnostic guidelines for Alzheimer's disease. Alzheimers Dement. 2011;7:280-292.

22. Shi F, Liu B, Zhou Y, Yu C, Jiang T. Hippocampal volume and asymmetry in mild cognitive impairment and Alzheimer's disease: meta-analyses of MRI studies. Hippocampus. 2009;19:1055-1064

23. Yushkevich PA, Pluta JB, Wang H, et al. Automated volumetry and regional thickness analysis of hippocampal subfields and medial temporal cortical structures in mild cognitive impairment. Hum Brain Mapp. September 2, 2014 [Epub ahead of print].

24. Da X, Toledo JB, Zee J, et al. Integration and relative value of biomarkers for prediction of $\mathrm{MCI}$ to $\mathrm{AD}$ progression: spatial patterns of brain atrophy, cognitive scores, APOE genotype and CSF biomarkers. Neuroimage Clin. 2014;4:164-173.

25. Minoshima S, Giordani B, Berent S, Frey KA, Foster NL, Kuhl DE. Metabolic reduction in the posterior cingulate cortex in very early Alzheimer's disease. Ann Neurol. 1997;42:85-94.

26. Kadir A, Almkvist O, Forsberg A, et al. Dynamic changes in PET amyloid and FDG imaging at different stages of Alzheimer's disease. Neurobiol Aging. 2012;33:198 e1-e14.

27. Dukart J, Mueller K, Villringer A, et al. Relationship between imaging biomarkers, age, progression and symptom severity in Alzheimer's disease. Neuroimage Clin. 2013;3:84-94.

28. Jack CR Jr, Knopman DS, Jagust WJ, et al. Tracking pathophysiological processes in Alzheimer's disease: an updated hypothetical model of dynamic biomarkers. Lancet Neurol. 2013;12:207-216.

29. Drzezga A, Lautenschlager N, Siebner H, et al. Cerebral metabolic changes accompanying conversion of mild cognitive impairment into Alzheimer's disease: a PET follow-up study. Eur J Nucl Med Mol Imaging. 2003;30:1104-1113.

30. Teune LK, Bartels AL, de Jong BM, et al. Typical cerebral metabolic patterns in neurodegenerative brain diseases. Mov Disord. 2010;25:2395-2404.

31. Jagust W, Reed B, Mungas D, Ellis W, Decarli C. What does fluorodeoxyglucose PET imaging add to a clinical diagnosis of dementia? Neurology. 2007;69:871-877.

32. Laforce R Jr, Buteau JP, Paquet N, Verret L, Houde M, Bouchard RW. The value of PET in mild cognitive impairment, typical and atypical/unclear dementias: a retrospective memory clinic study. Am J Alzheimers Dis Other Demen. 2010; 25:324-332.

33. Vandenberghe R, Adamczuk K, Dupont P, Laere KV, Chetelat G. Amyloid PET in clinical practice: its place in the multidimensional space of Alzheimer's disease. Neuroimage Clin. 2013;2:497-511.

34. Wolk DA, Price JC, Saxton JA, et al. Amyloid imaging in mild cognitive impairment subtypes. Ann Neurol. 2009;65:557-568.

35. Jack CR Jr, Wiste HJ, Vemuri P, et al. Brain beta-amyloid measures and magnetic resonance imaging atrophy both predict time-to-progression from mild cognitive impairment to Alzheimer's disease. Brain. 2010;133:3336-3348.

36. Koedam EL, Lauffer V, van der Vlies AE, van der Flier WM, Scheltens P, Pijnenburg YA. Early-versus late-onset Alzheimer's disease: more than age alone. J Alzheimers Dis. 2010;19:1401-1408.

37. Wolk DA. Amyloid imaging in atypical presentations of Alzheimer's disease. Curr Neurol Neurosci Rep. 2013;13:412.

38. Migliaccio R, Agosta F, Rascovsky K, et al. Clinical syndromes associated with posterior atrophy: early age at onset AD spectrum. Neurology. 2009;73:1571-1578.

39. Wolk DA, Price JC, Madeira C, et al. Amyloid imaging in dementias with atypical presentation. Alzheimers Dement. 2012;8:389-398. 
40. Pereira JM, Williams GB, Acosta-Cabronero J, et al. Atrophy patterns in histologic vs clinical groupings of frontotemporal lobar degeneration. Neurology. 2009;72:1653-1660.

41. Lindberg O, Ostberg P, Zandbelt BB, et al. Cortical morphometric subclassification of frontotemporal lobar degeneration. AJNR. 2009;30:1233-1239.

42. Rohrer JD, Geser F, Zhou J, et al. TDP-43 subtypes are associated with distinct atrophy patterns in frontotemporal dementia. Neurology. 2010;75:2204-2211.

43. Jeong Y, Cho SS, Park JM, et al. ${ }^{18} \mathrm{~F}-\mathrm{FDG}$ PET findings in frontotemporal dementia: an SPM analysis of 29 patients. J Nucl Med. 2005;46:233-239.

44. Nestor PJ, Fryer TD, Hodges JR. Declarative memory impairments in Alzheimer's disease and semantic dementia. Neuroimage. 2006;30:1010-1020.

45. Poljansky S, Ibach B, Hirschberger B, et al. A visual $\left[{ }^{18} \mathrm{~F}\right]$ FDG-PET rating scale for the differential diagnosis of frontotemporal lobar degeneration. Eur Arch Psychiatry Clin Neurosci. 2011;261:433-446.

46. Villemagne VL, Ong K, Mulligan RS, et al. Amyloid imaging with ${ }^{18} \mathrm{~F}$-florbetaben in Alzheimer disease and other dementias. J Nucl Med. 2011;52:1210-1217.

47. Rabinovici GD, Furst AJ, O'Neil JP, et al. ${ }^{11} \mathrm{C}$-PIB PET imaging in Alzheimer disease and frontotemporal lobar degeneration. Neurology. 2007;68:1205-1212.

48. Kitagaki H, Hirono N, Ishii K, Mori E. Corticobasal degeneration: evaluation of cortical atrophy by means of hemispheric surface display generated with MR images. Radiology. 2000;216:31-38.

49. Wadia PM, Lang AE. The many faces of corticobasal degeneration. Parkinsonism Relat Disord. 2007;13(suppl 3):S336-S340.

50. McKeith IG. Consensus guidelines for the clinical and pathologic diagnosis of dementia with Lewy bodies (DLB): report of the Consortium on DLB International Workshop. J Alzheimers Dis. 2006;9:417-423.

51. Edison P, Rowe CC, Rinne JO, et al. Amyloid load in Parkinson's disease dementia and Lewy body dementia measured with $\left[{ }^{11} \mathrm{C}\right] \mathrm{PIB}$ positron emission tomography. J Neurol Neurosurg Psychiatry. 2008;79:1331-1338.

52. Whitwell JL, Weigand SD, Shiung MM, et al. Focal atrophy in dementia with Lewy bodies on MRI: a distinct pattern from Alzheimer's disease. Brain. 2007;130:708-719.

53. Gilman S, Koeppe RA, Little R, et al. Differentiation of Alzheimer's disease from dementia with Lewy bodies utilizing positron emission tomography with $\left[{ }^{18}\right.$ F]fluorodeoxyglucose and neuropsychological testing. Exp Neurol. 2005;191 (suppl 1):S95-S103.

54. Papathanasiou ND, Boutsiadis A, Dickson J, Bomanji JB. Diagnostic accuracy of [I-123]-FP-CIT (DaTSCAN) in dementia with Lewy bodies: a meta-analysis of published studies. Parkinsonism Relat Disord. 2012;18:225-229.

55. Du AT, Schuff N, Kramer JH, et al. Different regional patterns of cortical thinning in Alzheimer's disease and frontotemporal dementia. Brain. 2007;130: 1159-1166.

56. Rohrer JD, Warren JD, Modat M, et al. Patterns of cortical thinning in the language variants of frontotemporal lobar degeneration. Neurology. 2009;72: $1562-1569$

57. Minoshima S, Foster NL, Sima AA, Frey KA, Albin RL, Kuhl DE. Alzheimer's disease versus dementia with Lewy bodies: cerebral metabolic distinction with autopsy confirmation. Ann Neurol. 2001;50:358-365.

58. Benson DF, Kuhl DE, Hawkins RA, Phelps ME, Cummings JL, Tsai SY. The fluorodeoxyglucose ${ }^{18} \mathrm{~F}$ scan in Alzheimer's disease and multi-infarct dementia. Arch Neurol. 1983;40:711-714.

59. Tripathi M, Tripathi M, Damle N, et al. Differential diagnosis of neurodegenerative dementias using metabolic phenotypes on F-18 FDG PET/CT. Neuroradiol J. 2014;27:13-21.

60. Perani D, Schillaci O, Padovani A, et al. A survey of FDG- and amyloid-PET imaging in dementia and GRADE analysis. BioMed Res Int. 2014;2014:785039.

61. Matias-Guiu JA, Cabrera-Martin MN, Garcia-Ramos R, et al. Evaluation of the new consensus criteria for the diagnosis of primary progressive aphasia using fluorodeoxyglucose positron emission tomography. Dement Geriatr Cogn Disord. 2014;38:147-152.

62. Prins ND, van der Flier WM, Brashear HR, et al. Predictors of progression from mild cognitive impairment to dementia in the placebo-arm of a clinical trial population. J Alzheimers Dis. 2013;36:79-85.
63. Prestia A, Caroli A, Herholz K, et al. Diagnostic accuracy of markers for prodromal Alzheimer's disease in independent clinical series. Alzheimers Dement. 2013;9:677-686.

64. Jack CR Jr, Petersen RC, Xu YC, et al. Prediction of AD with MRI-based hippocampal volume in mild cognitive impairment. Neurology. 1999;52:1397-1403.

65. Jack CR Jr, Shiung MM, Weigand SD, et al. Brain atrophy rates predict subsequent clinical conversion in normal elderly and amnestic MCI. Neurology. 2005; $65: 1227-1231$

66. Nestor SM, Rupsingh R, Borrie M, et al. Ventricular enlargement as a possible measure of Alzheimer's disease progression validated using the Alzheimer's disease neuroimaging initiative database. Brain. 2008;131:2443-2454.

67. Josephs KA Jr, Whitwell JL, Weigand SD, et al. Predicting functional decline in behavioural variant frontotemporal dementia. Brain. 2011;134:432-448.

68. Nedelska Z, Ferman TJ, Boeve BF, et al. Pattern of brain atrophy rates in autopsy-confirmed dementia with Lewy bodies. Neurobiol Aging. July 15, 2014 [Epub ahead of print].

69. Fujishiro H, Iseki E, Kasanuki K, et al. A follow up study of non-demented patients with primary visual cortical hypometabolism: prodromal dementia with Lewy bodies. J Neurol Sci. 2013;334:48-54.

70. Jacova C, Hsiung GY, Tawankanjanachot I, et al. Anterior brain glucose hypometabolism predates dementia in progranulin mutation carriers. Neurology. 2013;81:1322-1331.

71. Lowe VJ, Weigand SD, Senjem ML, et al. Association of hypometabolism and amyloid levels in aging, normal subjects. Neurology. 2014;82:1959-1967.

72. Rowe CC, Bourgeat P, Ellis KA, et al. Predicting Alzheimer disease with betaamyloid imaging: results from the Australian imaging, biomarkers, and lifestyle study of ageing. Ann Neurol. 2013;74:905-913.

73. Roe CM, Fagan AM, Grant EA, et al. Amyloid imaging and CSF biomarkers in predicting cognitive impairment up to 7.5 years later. Neurology. 2013;80:17841791.

74. Shaffer JL, Petrella JR, Sheldon FC, et al. Predicting cognitive decline in subjects at risk for Alzheimer disease by using combined cerebrospinal fluid, MR imaging, and PET biomarkers. Radiology. 2013;266:583-591.

75. Zhang D, Shen D. Multi-modal multi-task learning for joint prediction of multiple regression and classification variables in Alzheimer's disease. Neuroimage. 2012;59:895-907.

76. Gross AL, Manly JJ, Pa J, et al. Cortical signatures of cognition and their relationship to Alzheimer's disease. Brain Imaging Behav. 2012;6:584-598.

77. Westman E, Muehlboeck JS, Simmons A. Combining MRI and CSF measures for classification of Alzheimer's disease and prediction of mild cognitive impairment conversion. Neuroimage. 2012;62:229-238.

78. Nettiksimmons J, Harvey D, Brewer J, et al. Subtypes based on cerebrospinal fluid and magnetic resonance imaging markers in normal elderly predict cognitive decline. Neurobiol Aging. 2010;31:1419-1428.

79. Johnson NA, Jahng GH, Weiner MW, et al. Pattern of cerebral hypoperfusion in Alzheimer disease and mild cognitive impairment measured with arterial spinlabeling MR imaging: initial experience. Radiology. 2005;234:851-859.

80. Hu WT, Wang Z, Lee VM, Trojanowski JQ, Detre JA, Grossman M. Distinct cerebral perfusion patterns in FTLD and AD. Neurology. 2010;75:881-888.

81. Celone KA, Calhoun VD, Dickerson BC, et al. Alterations in memory networks in mild cognitive impairment and Alzheimer's disease: an independent component analysis. J Neurosci. 2006;26:10222-10231.

82. Thomas JB, Brier MR, Bateman RJ, et al. Functional connectivity in autosomal dominant and late-onset Alzheimer disease. JAMA Neurol. 2014;71:1111-1122.

83. Fodero-Tavoletti MT, Okamura N, Furumoto S, et al. ${ }^{18} \mathrm{~F}-\mathrm{THK} 523$ : a novel in vivo tau imaging ligand for Alzheimer's disease. Brain. 2011;134:1089-1100.

84. Arriagada PV, Growdon JH, Hedley-Whyte ET, Hyman BT. Neurofibrillary tangles but not senile plaques parallel duration and severity of Alzheimer's disease. Neurology. 1992;42:631-639.

85. Kreisl WC, Lyoo $\mathrm{CH}$, McGwier $\mathrm{M}$, et al. In vivo radioligand binding to translocator protein correlates with severity of Alzheimer's disease. Brain. 2013; 136:2228-2238. 